\title{
Compromiso epidural espinal como presentación de los linfomas no hodgkinianos: aportación de 6 casos
}

\author{
A. SIMIELE NARVARTE, N. GÓMEZ RODRÍGUEZ1, F. NOVOA SANJURJO \\ Servicios de Hematología y de ${ }^{1}$ Reumatología del Centro Médico POVISA. Vigo. \\ Pontevedra
}

NON-HODGKING'S LYMPHOMA PRESENTING WITH EPIDURAL SPINAL INVOLVEMENT: A REPORT OF SIX CASES

\begin{abstract}
RESUMEN
La compresión mielorradicular es infrecuente en el curso de los linfomas no hodgkinianos, generalmente ocurre por afección extradural y rara vez constituye su manifestación inicial. Aunque puede implicarse cualquier segmento espinal, se ha descrito cierto predominio en la región dorsal. En la mayoría de los casos se trata de linfomas de inmunofenotipo B de alto grado y su comportamiento es agresivo.

En el presente trabajo aportamos los casos de seis adultos, cinco mujeres y un varón, en quienes las complicaciones neurológicas espinales constituyeron la primera manifestación de un linfoma no hodgkiniano y procedemos a revisar la literatura sobre estos procesos.
\end{abstract}

PALABRAS CLAVE: Linfoma no Hodgkin. Complicaciones neurológicas. Espacio epidural. Columna vertebral.

\begin{abstract}
Spinal cord compression and radicular involvement are infrequent events in the natural history of non-Hodgkin's lymphoma, which usually are secondary to invasion of spinal extradural space, and rarely are the presenting manifestations of this condition. Thoracic segment is predominantly affected, but any spinal region can be affected. Histologically, the spinal tumour are frequently a high-grade B lymphoma with aggressive behaviour.

The aim of this work was to describe six new cases of non-Hodgkin's lymphoma in adults (five woman and one men), presenting with spinal neurological complications. The medical literature about this entity are revised.
\end{abstract}

KEY WORDS: Non-Hodgkin's lymphoma. Neurological complications. Epidural space. Spine.

Simiele Narvarte A, Gómez, Rodríguez N, Novoa Sanjurjo F. Compromiso epidural espinal como presentación de los linfomas no hodgkinianos: aportación de 6 casos. An Med Interna (Madrid) 2003; 20: 466-469.

\section{INTRODUCCIÓN}

En su historia natural, el $5 \%$ de los tumores malignos, sobre todo los sólidos, originan complicaciones neurológicas espinales que, en las dos terceras partes de los casos, afectan sólo a un nivel vertebral (1). En los linfomas no hodgkinianos (LNH) se estima que este tipo de compromiso es raro, y se ha reseñado, según las series, entre el $0,1 \%$ y el 6,5\%, siendo más proclives a ello los LNH difusos de células grandes (2-4). Las formas más excepcionales de afección medular son las intraparenquimatosas $(5,6)$ y la compresión extrínseca por una hemorragia originada en los vasos de las leptomeninges (7). El compromiso a partir de la infiltración epidural constituye el mecanismo más habitual en las complicaciones neurológicas espinales de los LNH (2-4, 8-17). En nuestro país, han sido muy pocos los pacientes descritos con compromiso mielorradicular por los LNH (18-21), por lo que hemos considerado de interés aportar 6 nuevos casos y revisar la información disponible acerca de sus manifestaciones clínicas, diagnóstico por la imagen, tratamiento y pronóstico.

\section{CASOS APORTADOS}

Caso 1: Mujer de 30 años, sin antecedentes personales ni familiares de interés, que fue ingresada por un cuadro insidioso caracterizado por dorsalgias de ritmo inflamatorio, disnea progresiva, sudoración nocturna y, en las últimas dos semanas, pérdida de fuerza en las extremidades inferiores que dificultaba la deambulación.

En la exploración destacaba la semiología de derrame pleural derecho, una paraparesia flácida en las extremidades inferiores e hipoestesia bajo el dermatoma D6. No se palpaban adenopatías periféricas, visceromegalias ni masas abdominales. No se identificaron fiebre ni lesiones cutáneas.

Datos de laboratorio: discreta anemia normocítica normocrómica (Hb: 108g/L), elevación de la LDH (1.151 UI/L), $\beta$-2-microglobulina $(2,68 \mu \mathrm{g} / \mathrm{L}$; normal hasta 2,4$)$ y VSG $\left(72 \mathrm{~mm} / 1^{\mathrm{a}} \mathrm{h}\right)$. Las siguientes determinaciones fueron normales o negativas: transaminasas, fosfatasa alcalina, calcio, fósforo, sodio, potasio, cloro, ácido úrico, colesterol total, bilirrubina, estudio completo de coagulación, marcadores de la hepatitis B y C así como anticuerpos frente al virus de la inmunodeficiencia humana (VIH) tipos I y II.

En la radiografía simple de tórax se apreció un derrame pleural derecho no asociado a ensanchamiento mediastínico. Se practicó una

Trabajo aceptado: 12 de enero de 2003 
TAC toracoabdominal y de columna dorsal en las que se objetivó una masa paraespinal adyacente a D5 y D6 así como una tumoración epidural a dichos niveles (Fig. 1). Se procedió a una laminectomía descompresiva desde D5 a D7 y a la resección parcial de la tumoración intrarraquídea. Su estudio anatomopatológico fue compatible con un LNH linfoblástico difuso de célula grande B. La biopsia de médula ósea no demostró infiltración neoplásica.

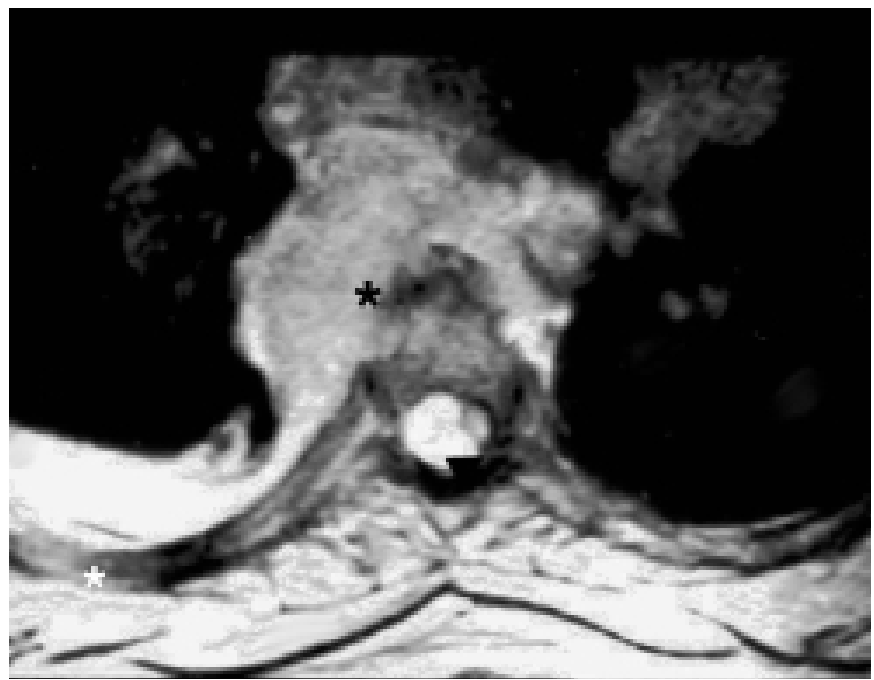

Fig. 1. Corte axial de tomografía computarizada a la altura de la quinta vértebra dorsal de la primera de las pacientes descritas. El linfoma no Hodgkin $(*)$ erosionaba el cuerpo vertebral e invadía el interior del canal medular (punta de flecha).

El tratamiento se completó con radioterapia local y 6 ciclos de quimioterapia (protocolo CHOP). La evolución fue favorable y se alcanzó la remisión completa. En la última revisión, siete años después de finalizado el tratamiento, la paciente permanece asintomática.

Caso 2: Mujer de 60 años que ingresó por dolor lumbar de ritmo inflamatorio con irradiación ciatálgica atípica bilateral y, durante las dos semanas previas a la consulta, dificultad rápidamente progresiva para la deambulación. En la exploración destacaba la existencia de una paraparesia flácida y retención urinaria por vejiga neurógena.

Entre los datos de laboratorio destacaban una discreta anemia microcítica (Hb: 115g/L Hcto: 32,3 L/L VCM: $78 \mathrm{fl}$ ). Los valores de VSG, proteína C reactiva, y LDH fueron normales. Los anticuerpos frente a los virus de la hepatitis B y C y HIV (tipos I y II) fueron negativos.

En el estudio de imagen (TAC toracoabdominal y de columna dorsolumbar) se demostró la presencia de una masa epidural desde L1 hasta L3 que comprometía las caras posterior y lateral del saco dural. Se procedió a realizar una laminectomía descompresiva desde L1 a L3, con exéresis del tumor. El estudio histológico demostró un LNH centrocítico-centroblástico difuso de células B. En la biopsia de medula ósea no se evidenció infiltración por linfoma.

Se trató con radioterapia local y con quimioterapia según protocolo CHOP. Fue controlada en nuestro centro durante los siguientes tres años sin que hubiese evidencia de recidiva.

Caso 3: Mujer de 65 años, sin antecedentes personales de interés, que ingresó a causa de dolor abdominal no cólico irradiado a región dorsolumbar, iniciado dos semanas antes y al que, en las últimas 72 horas, se había añadido dificultad para la deambulación.

En la exploración destacaba una paraparesia flácida y una vejiga neurógena. La resonancia magnética dorsolumbar demostró alteraciones de la señal, sugestivas de proceso infiltrativo tumoral en los cuerpos vertebrales desde D7 hasta D10. La TAC toracoabdominal demostró nódulos pulmonares bilaterales, derrame pericárdico y esplenomegalia sin lesiones ocupantes de espacio.
Entre los datos de laboratorio destacaban una anemia normocítica no hemolítica (Hb: $93 \mathrm{~g} / \mathrm{L}$ Hcto: $0.28 \mathrm{~L} / \mathrm{L}$ VCM: $88 \mathrm{fl})$, elevación de la LDH (545 U/L). VSG: $45 \mathrm{~mm}, \beta$-2-microglobulina: 3,0 $\mu \mathrm{g} / \mathrm{L}$. Las serologías para hepatitis B y C y VIH fueron negativas. Biopsia de medula ósea sin evidencia de infiltración.

El tratamiento consistió en una laminectomía descompresiva desde D7 hasta D10 con exéresis de la tumoración epidural, cuyo estudio histológico demostró un LNH de células grandes B. Se completó con radioterapia local y quimioterapia (CHOP). Sólo se obtuvo una remisión parcial, sin mejoría de los déficit neurológicos. Pese al tratamiento, el LNH progresó apareciendo signos de afección sistémica. La paciente fue exitus a los 18 meses del diagnóstico.

Caso 4: Varón de 31 años, remitido al servicio de neurología por paraplejia de instauración subaguda ( 2 meses), pérdida ponderal, anorexia y sudoración nocturna.

Entre los datos de laboratorio cabía reseñar una anemia normocrómica normocítica (Hb: $89 \mathrm{~g} / \mathrm{L}$, hematocrito: 0,26 L/L, VCM: 86 fl), LDH: $402 \mathrm{U} / \mathrm{L}, \beta-2$-microglobulina $(3,43 \mu \mathrm{g} / \mathrm{L})$. VSG: 87 $\mathrm{mm} / 1^{\mathrm{a} h}$. Marcadores de la hepatitis B y C negativos. Anticuerpos frente a VIH tipos I y II negativos. Biopsia de medula ósea sin signos de infiltración neoplásica.

La resonancia magnética dorsolumbar demostró una tumoración epidural a la altura de D8-D9. En la TAC toracoabdominal se observaron hepatoesplenomegalia, adenopatías peripancreáticas y nódulos pulmonares bilaterales. El tratamiento incluyó laminectomía descompresiva (D8 y D9) con resección completa del tumor, cuya histología fue compatible con LNH de células grandes B. Adicionalmente se aplicó radioterapia local y se administraron 4 ciclos de quimioterapia (CHOP), obteniéndose una remisión parcial, aunque el enfermo se mantuvo parapléjico. El paciente falleció 12 meses después del diagnóstico a causa de la afectación sistémica por el LNH.

Caso 5: Mujer de 60 años, internada por lumbalgia inflamatoria asociada a ciatalgia atípica bilateral de 5 meses de evolución.

En la TAC toracoabdominal se apreciaron lesiones lumbares a la altura de L3, L4 y L5. En los análisis llamaba la atención la presencia de una anemia normocrómica normocítica con $\mathrm{Hb}$ de $98 \mathrm{~g} / \mathrm{L}$, hematocrito (0,30 L/L), VCM: 87 fl. VSG: $103 \mathrm{~mm} / 1^{\text {a h} . ~ L D H: ~} 1,110$ U/L. $\beta$-2-microglobulina: 3,5 $\mu \mathrm{g} / \mathrm{L}$. Los anticuerpos frente al VIH asî como los marcadores de la hepatitis $\mathrm{B}$ y $\mathrm{C}$ fueron negativos.

Se procedió a una laminectomía desde L3 hasta L5 y se biopsió la tumoración epidural. El estudio anatomopatológico fue compatible con un LNH centroblástico. No se demostró infiltración por el linfoma en la biopsia de la médula ósea. A pesar de la quimioterapia (protocolo CHOP), la evolución fue desfavorable y la enferma falleció 18 meses después del diagnóstico.

Caso 6: Mujer de 28 años que consultó por malestar general y lumbalgia de ritmo inflamatorio de 5 meses de evolución. La exploración física demostró presencia de hepatosplenomegalia, derrame pleural derecho, hipotrofia muscular generalizada con dificultad en la deambulacion. La TAC permitió objetivar una tumoración frente a D8-D10, con compromiso de los cuerpos vertebrales, asociada a hepatoesplenomegalia y derrame pleural derecho. En los análisis se demostró una anemia normocítica (Hb: $93 \mathrm{~g} / \mathrm{L}), \mathrm{LDH}(371 \mathrm{U} / \mathrm{L}), \mathrm{VSG}\left(76 \mathrm{~mm} / \mathrm{1}^{\mathrm{a}} \mathrm{h}\right), \beta$ 2-microglobulina $(3,5 \mu \mathrm{g} / \mathrm{ml})$. Los anticuerpos frente a HIV I y II asî como los marcadores de hepatitis B y C fueron negativos. La biopsia de medula ósea no demostró signos de infiltración tumoral.

Se efectuó una laminectomía descompresiva desde D8 hasta D10, siendo el diagnóstico anatomopatológico de las muestras tomadas de la masa epidural compatible con LNH centroblástico. No hubo respuesta a la quimioterapia y la enferma falleció a los tres meses del diagnóstico.

\section{DISCUSIÓN}

Aunque pueden aparecer a cualquier edad y con incidencia similar para ambos sexos, los linfomas malignos son la neoplasia más frecuente entre los adultos de 20 a 40 años. Tras la 
irrupción del síndrome de inmunodeficiencia adquirida (SIDA), su incidencia se ha incrementado. Excluyendo a dichos pacientes, la afectación del sistema nervioso central por LNH es una de las más raras localizaciones extraganglionares. Aunque, en alguna serie de laminectomías descompresivas por compromiso mielorradicular, los LNH supusieron el $15 \%$ de las etiologías subyacentes, puede afirmarse que, en general, las complicaciones neurológicas espinales constituyen la primera manifestación de esta neoplasia en un pequeño porcentaje de casos que oscila entre el $0,1 \%$ y el $6,5 \%(2-4)$, con un promedio del $2-3 \%$, tanto en adultos $(3,4,6)$ como en las series pediátricas (14). El efecto masa a partir de la afectación extradural constituye el mecanismo habitual, aunque rara vez se ha descrito la compresión extrínseca por hematomas epidurales secundarios (7). Estos, a su vez, podrían desencadenarse por lesión de las paredes vasculares y estar facilitados por otros factores como la quimioterapia, trombopenia o alteraciones de la coagulación. Como ocurre con la mayoría de las neoplasias malignas, la invasión epidural de los LNH suele producirse a partir de los tejidos adyacentes, en especial desde la porción posterior de los cuerpos vertebrales, ya que el ligamento vertebral común posterior es una barrera relativamente débil comparada con los ligamentos amarillos y las plataformas cartilaginosas que delimitan los espacios discales. El plexo venoso de Batson constituye una vía fácil de diseminación hacia el interior del canal medular (22). Tras la consecución de una remisión completa con el tratamiento, el compromiso mielorradicular por afectación epidural puede suponer la revelación de una recidiva del LNH (23). La afección intramedular de un LNH resulta excepcional $(5,6,16)$ y en los pocos casos reseñados en la literatura predomina la localización cervical seguida de la dorsal (16).

Entre los LNH responsables de complicaciones neurológicas espinales predominan los de alto grado e inmunofenotipo B, sobre todo los linfomas difusos de células grandes $(4,13,19,24)$. En un tercio de los pacientes la extensión leptomeníngea del LNH implica distintos niveles simultáneamente, pero, en la mayoría de los casos, se objetivan lesiones únicas (1).

El cortejo sintomático se instaura habitualmente de forma subaguda. Suelen coexistir raquialgias localizadas de ritmo inflamatorio (50-90\% pacientes) y debilidad en las extremidades inferiores cuya intensidad va desde una leve paraparesia hasta una paraplejia, con o sin trastornos esfinterianos $(13,19,24,25)$. Se han descrito casos excepcionales en los que la expresión clínica emuló la de una estenosis de canal medu- lar (26), una radiculopatía (20) o una mielopatía cervical espondiloartrósica (27), así como asociaciones con polirradiculoneuritis de Guillain-Barré (28) o con infecciones oportunistas (29).

La resonancia magnética constituye el medio de diagnóstico por la imagen de elección debido a su elevada sensibilidad y a su capacidad para delimitar la extensión del LNH $(11,30)$, especialmente cuando se utiliza contraste paramagnético (31). Las radiografías simples, aunque pueden mostrar aumento del volumen y densidad de partes blandas paravertebrales o lesiones óseas vertebrales, muchas veces son normales hasta fases avanzadas de la enfermedad $(8,10,12,14)$. La tomografía computarizada también permite identificar masas, por lo general de localización extradural y detectar lesiones óseas vertebrales, aunque tiene menor sensibilidad para los raros casos de afección intradural e intramedular.

Como en cualquier paciente con semiología de compromiso mielorradicular, la etiología debe establecerse en el menor plazo posible, procediendo de inmediato a liberar el segmento afectado, ya que el pronóstico neurológico depende de la intensidad y de la duración de la compresión. Por otra parte, los hallazgos de la tomografía computarizada o de la resonancia magnética no son suficientes para precisar la naturaleza de la masa tumoral. Así, en el acto quirúrgico, además conseguir la descompresión espinal, se obtendrán muestras de tejido para estudio anatomopatológico, a partir del que se establecerá el diagnóstico de LNH $(3,4,25)$. Aquellos enfermos ya diagnosticados de LNH que en su curso clínico presenten recidivas con compromiso mielorradicular pueden también beneficiarse de la laminectomía descompresiva. No obstante, cuando los déficit neurológicos progresan lentamente, la combinación de radioterapia y quimioterapia puede bastar para obtener la regresión del tumor. La quimioterapia, habitualmente con la pauta CHOP (ciclofosfamida, adriamicina, vincristina y prednisona), bien de forma aislada o en combinación, constituye la base del tratamiento de los LNH con compromiso mielorradicular $(4,13,16,19)$. Aunque los LNH son muy sensibles a la radioterapia, su empleo aislado se sigue de una elevada tasa de recidivas, siendo aconsejable la asociación con poliquimioterapia.

El diagnóstico tardío, la localización del LNH en la región cervical y porción superior del segmento dorsal, la rápida progresión del déficit neurológico y la aparición de tetraplejia, así como los pacientes con SIDA, se asocian con un peor pronóstico (19).

\section{Bibliografía}

1. Chamberlain M C, Kormanik P A. Epidural spinal cord compression: a single institution's retrospective experience. Neuro Oncol 1999; 1: 1203.

2. Correale J, Monteverde D A, Bueri J A, Reich E G. Peripheral nervous system and spinal cord involvement in lymphoma. Spine 1991; 16: 377-9.

3. Lakshmaiah K C, Lokanat D, Suresh T M, Babu K G, Ramesh C, Rao C $\mathrm{R}$, et al. Spinal cord compression by primary non-Hodgkin's lymphoma. Indian J Cancer 1995; 32: 81-4.

4. Salvati M, Cervoni L, Artico M, Raco A, Cappetta P, Delfíni. Primary spinal epidural non-Hodgkin's lymphomas: a clinical study. Surg Neurol 1996; 46: 339-43

5. Herida M, Khuong MA, De Broucker T, Henin D, Mecchali D. Lymphome primitif du système nerveux central a localisation medullaire revelatrice chez un patient infecté par le virus de l'inmunodeficiencie humaine. Ann Med Inter (París) 1993; 144: 64-6.

6. Drouet A, Caminade V, Guilloton L, Brunot J, Crozes P, Ribot C. Lymphome intramedullaire primitif (LIMP) en dehors de l'infection par le VIH. Aspects en IRM. Rev Neurol (París) 1999; 155: 1074-8.

7. Mastronardi L, Carletti S, Frondizi D, Spera C, Maira G. Cervical spontaneous epidural hematoma as a complication of non-Hodgkin's lymphoma. Eur Spine J 1996; 5: 268-71. 
8. Lyons MK, O-Neill BP, Marsh WR, Kurtin PJ. Primary spinal epidural non-Hodgkin's lymphoma: report of eight patients and review of the literature. Neurosurgery 1992; 30: 675-80.

9. Rathmell AJ, Gospodarowicz MK, Sutcliffe SB, Clark RM. Localized extradural lymphoma: survival, relapse pattern and functional outcome. The Princess Margaret Hospital Lymphoma Group. Radiother Oncol 1992; 24: 14-20.

10. Perry JR, Deodhare SS, Bilbao JM, Murray D, Muller P. The significance of spinal cord compression as the initial manifestation of lymphoma. Neurosurgery 1993; 32: 157-162.

11. Boukobza M, Mazel C, Touboul E. Primary vertebral and spinal epidural non-Hodgkin's lymphoma with spinal cord compression. Neuroradiology 1996; 38: 333-7.

12. Lim CC, Chong BK. Spinal epidural non-Hodgkin's lymphoma: case reports of three patients presenting with spinal cord compression. Singapore Med J 1996; 37: 497-500.

13. Raco A, Cervoni L, Salvati M, Delfini R. Primary spinal epidural nonHodgkin's lymphomas in childhood: a review of 6 cases. Acta Neurochir 1997; 139: 526-8.

14. Mora J, Wollner N. Primary epidural non-Hodgkin lymphoma: spinal cord compresión síndrome as the initial presentation in childhood nonHodgkin's lymphoma. Med Pediatric Oncol 1999; 32: 102-5.

15. McDonald AC, Nicoll JA, Rampling RP. Non-Hodgkin's lymphoma presenting with spinal cord compression: a clinicopathological review of 25 cases. Eur J Cancer 2000; 36: 207-13

16. Pels H, Vogt I, Klockgether T, Schlegel U. Primary non-Hodgkin's of the spinal cord. Spine 2000; 25: 2262-4.

17. Ching HY, Horsman JM, Radstone CR, Hancock H, Timperley W R, Hancock BW. Non Hodgkin's lymphoma presenting with spinal involvement: the Sheffield Lymphoma Group experience (1970-2000). Int J Oncol 2001; 19: 149-56.

18. Amigo ML, Revilla R, Sánchez C, del Cañizo C, Caballero D, San Miguel JF. Compresión medular como forma de presentación de linfoma no hodgkiniano. Sangre 1995; 40: 505-7.

19. Vela D, Ribera JM, Florensa R, Arellano A, Vaquero M, Battle M, et al.
Compresión medular en los linfomas no hodgkinianos. Estudio de 10 pacientes. Med Clin (Barc) 1997; 109: 375-7

20. Pérez DA, Saiz RA, de Toledo M, Berbel A, Martínez A, Prieto S. Radiculopatía cervical como forma de presentación de un linfoma no Hodgkin. Rev Neurol 1998; 27: 91-93.

21. Paradas C, Márquez C, López J C. Linfoma intrarraquídeo. Neurología 1999; 14: 407.

22. Fujita T, Ueda Y, Kawahara N, Baba H, Tomita K. Local spread of metastatic vertebral tumors. Spine 1997; 22: 1905-12.

23. Laing R J, Jakubowski J, Kunkler I H, Hancock B W. Primary spinal presentation of non-Hodgkin`s lymphoma. A reappraisal of management and prognosis. Spine 1992; 17: 117-20.

24. Ching HY, Horsman JM, Radstone CR, Hancock H, Timperley WR, Hancock B W. Non-Hodgkin`s lymphoma presenting with spinal involvement: the Sheffield Lymphoma Group experience (1970-2000). Int J Oncol 2001; 19: 149-56.

25. Eeles RA, O`Brien P, Horwich A, Brada M. Non-Hodgkin`s lymphoma presenting with extradural spinal cord compression: functional outcome and survival. Br J Cancer 1991; 63: 126-9.

26. Travlos J, du Toit G. Primary spinal epidural lymphoma mimicking lumbar spinal stenosis. A case report. Spine 1991; 16: 277-379.

27. Caruso PA, Patel MR, Joseph J, et al. Primary intramedullary lymphoma of the spinal cord mimicking cervical spondylitic myelopathy. A J R 1998; 171: 526-7.

28. Guecueyener K, Keskil S, Baykaner MK, Bilir E, Oguz A, Ceviker N. Co-incidence of Guillain-Barré syndrome and spinal cord compression in non-Hodgkin lymphoma. Neuropediatrics 1994; 25: 36-8.

29. Moulignier A, Eliaszewics M, Mikol J, Polivka M, Thiebaut JB, Dupont B. Spinal cord compression due to concomitant primary lymphoma and Mycobacterium avium-intracellulare infection of the paravertebral muscles in an AIDS patient. J Clin Microbiol Infect Dis 1996; 15: 891-3.

30. Li MH, Holtaas S, Larsson EM. MR imaging of spinal lymphoma. Acta Radiol 1992; 33: 382-42.

31. Schild SE, Wharen REJr, Menke DM, Folger W N, Colon-Otero G. Primary lymphoma of the spinal cord. Mayo Clin Proc 1995; 70: 256-60. 